See discussions, stats, and author profiles for this publication at: https://www.researchgate.net/publication/264685633

\title{
The environmental controls that govern the end product of bacterial nitrate respiration
}

Article in Science · August 2014

CITATIONS

100

8 authors, including:

Beate Kraft

University of Southern Denmark

11 PUBLICATIONS 666 CITATIONS

SEE PROFILE

Martin G Klotz

Washington State University

151 PUBLICATIONS 7,080 CITATIONS

SEE PROFILE

Some of the authors of this publication are also working on these related projects:

Membrane transport View project

RV Meteor cruise M57-2 View project
READS

361

A Halina E Tegetmeyer

Max Planck Institute for Marine Microbiology

73 PUBLICATIONS 1,450 CITATIONS

SEE PROFILE

Timothy Ferdelman

Max Planck Institute for Marine Microbiology

294 PUBLICATIONS 8,557 CITATIONS

SEE PROFILE 
17. R. A. Schatzinger, J. F. Jordan, Reservoir Characterization: Recent Advances (American Association of Petroleum Geologists, Tulsa, OK, 1999).

\section{ACKNOWLEDGMENTS}

We thank G. Barthel, K. Hörmann, and M. Stöckl for technical assistance and B. Horsefield for helpful discussions. Funding was provided by Helmholtz Zentrum München and the DFG priority program SPP1319. Pyrotag sequencing data for community analyses are deposited at NCBI BioSample (www.ncbi.nlm.nih.gov/ biosample) under accession number SRP036082. Original data from FTICR-MS analyses are provided in the supplementary materials.
Supplementary Text

Figs. S1 to S6

Tables S1 to 55

References (18-38)

13 February 2014; accepted 26 June 2014

10.1126/science. 1252215

\section{The environmental controls that govern the end product of bacterial nitrate respiration}

\author{
Beate Kraft, ${ }^{1 *}$ Halina E. Tegetmeyer, ${ }^{1,2}$ Ritin Sharma, ${ }^{3,4}$ Martin G. Klotz, ${ }^{5,6}$ \\ Timothy G. Ferdelman, ${ }^{1}$ Robert L. Hettich, ${ }^{3,4}$ Jeanine S. Geelhoed, ${ }^{1,7}$ Marc Strous ${ }^{1,2,8}+$
}

In the biogeochemical nitrogen cycle, microbial respiration processes compete for nitrate as an electron acceptor. Denitrification converts nitrate into nitrogenous gas and thus removes fixed nitrogen from the biosphere, whereas ammonification converts nitrate into ammonium, which is directly reusable by primary producers. We combined multiple parallel long-term incubations of marine microbial nitrate-respiring communities with isotope labeling and metagenomics to unravel how specific environmental conditions select for either process. Microbial generation time, supply of nitrite relative to nitrate, and the carbon/nitrogen ratio were identified as key environmental controls that determine whether nitrite will be reduced to nitrogenous gas or ammonium. Our results define the microbial ecophysiology of a biogeochemical feedback loop that is key to global change, eutrophication, and wastewater treatment.

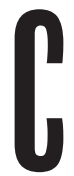
urrently, most fixed nitrogen in the biosphere originates from anthropogenic sources such as the industrial production of fertilizer ammonium. Uptake of fertilizer by crops is only $17 \%$ efficient, and 1 to $5 \%$ of fertilizer ammonium is converted biologically into nitrous oxide, a long-lived and powerful greenhouse gas (1). Microbial nitrification also converts a large portion of the fertilizer ammonium to nitrate in soil, where it subsequently runs off into surface waters and contributes to eutrophication in coastal zones. Nitrate emissions are partially remediated by denitrification in engineered environments such as wastewater treatment plants. If the end product of microbial nitrate reduction could be influenced by tuning environmental conditions, this would yield substantial ecological and eco-

${ }^{1}$ Max Planck Institute for Marine Microbiology, 28359 Bremen, Germany. ${ }^{2}$ Institute for Genome Research and Systems Biology, Center for Biotechnology, University of Bielefeld, 33615 Bielefeld, Germany. ${ }^{3}$ UT-ORNL Graduate School of Genome Science and Technology, University of Tennessee, Knoxville, TN 37996, USA. ${ }^{4}$ Chemical Science Division, Oak Ridge National Laboratory, Oak Ridge, TN 37830, USA. ${ }^{5}$ Department of Biological Sciences, University of North Carolina, Charlotte, NC 28223, USA. ${ }^{6}$ State Key Laboratory of Marine Environmental Science, Xiamen University, Xiamen 361005, China. ${ }^{7} \mathrm{NIOZ}$ Royal Netherlands Institute for Sea Research, 4401NT Yerseke, Netherlands. ${ }^{8}$ Department of Geoscience, University of Calgary, Calgary, Alberta T2N 1N4, Canada.

*Present address: Department of Organismic and Evolutionary Biology, Harvard University, Cambridge, MA 02138, USA †Corresponding author. E-mail: mstrous@ucalgary.ca nomic benefits for both natural and engineered systems.

Two microbial processes compete for nitrate as an electron acceptor: denitrification (including anammox) and ammonification (including dissimilatory nitrate reduction to ammonium). The carbon/nitrogen ratio (2-5), $\mathrm{pH}(5)$, nitrite versus nitrate concentration (5-7), soil sand content (5), availability of fermentable carbon compounds $(4,8)$, temperature $(7,9)$, and sulfide concentration (10-12) are potentially important environmental controls on this competition. The lack of consensus regarding which factors are most important, and in which environments, is likely due to the complex and highly variable structure of natural microbial communities.

To unravel the selective forces behind the ecological success of denitrification or ammonification, we subjected natural communities to specific environmental conditions in multiple parallel long-term incubations (13). The source community was from coastal, sandy tidal flat sediments that make substantial contributions to global denitrification and perform both denitrification and ammonification $(12,14)$. We did not aim to reproduce the sediment community in the laboratory; we simply used the sediment as a highly microbially diverse inoculum to enable the selection of optimally adapted nitratereducing communities. We performed 15 parallel anoxic incubations (Fig. 1 and table S1) with continuous substrate supply, which made it pos- sible to maintain the nitrate and/or nitrite concentrations in the in situ micromolar range ( $<0.5 \mu \mathrm{M}$ for nitrite, $<10 \mu \mathrm{M}$ for nitrate).

Denitrification and ammonification have two electron acceptors in common: nitrate and nitrite. In theory, the outcome of the competition could be most easily explained by which of these two compounds is supplied. In natural ecosystems, the relative supply of nitrate and nitrite is controlled by nitrification, a two-step process that can yield either compound as the end product. In our experiments with nitrate as the terminal electron acceptor, ammonification emerged as the prevalent pathway, whereas supply of nitrite resulted in denitrification prevalence (Fig. 1). Denitrification was always observed as the prevalent respiratory pathway when nitrite was supplied, even in the presence of fermentable substrates and sulfide, at low $\mathrm{pH}$ or at a reduced copper concentration. Thus, the supply of nitrite or nitrate was a key factor in the outcome of the competition between denitrification and ammonification. If elevated sulfide concentrations or changes in $\mathrm{pH}$ decrease the rate of nitrite production relative to the rate of nitrate production $(15,16)$, this would therefore favor ammonification over denitrification, as was observed in some previous studies $(5,12)$.

We further investigated the apparent success of ammonification with nitrate as the electron acceptor in a 400-day chemostat incubation. In a chemostat, the growth rate (or generation time) of the cultivated bacteria is controlled by the applied dilution rate, enabling us to test whether this factor affected the outcome of the competition in any way. The average in situ generation time of the sampled community was estimated at $\sim 0.4$ days, a value derived from a metagenome of the tidal flat community (17). During the 400day incubation, the generation time was varied between 1.0 and 3.4 days and the nitrite and nitrate concentrations always remained in the low micromolar range ( $<0.5 \mu \mathrm{M}$ for nitrite, $<10 \mu \mathrm{M}$ for nitrate). The generation time strongly affected the outcome of the competition for nitrate (Fig. 2). As shown by mass balancing, denitrification prevailed at short generation times, whereas ammonification was most successful at long generation times, with a tipping point detected at a generation time of $~ 1.7$ days (Fig. 2). After 185 days, we increased the generation time in the chemostat from 1.0 to 1.7 days and ammonification slowly became dominant; however, after day 230, denitrification regained prevalence. Ammonification only recovered after the generation time was further increased to 3.4 days after day 240 .

To determine the mechanisms responsible for the observed selective effect of microbial generation time, we characterized the selected 
Fig. 1. Fate of nitrite or nitrate in $\mathbf{1 5}$ anoxic incubations with continuous substrate supply. All incubations (except one) received nitrite as the main electron acceptor and exhibited denitrification. Only a single incubation (green circles, top right panel) received nitrate and exhibited ammonification. For medium composition, see table S1. Shaded areas indicate the composition of nitrogen compounds in the inflowing medium (bronze denotes nitrate or nitrite; apricot denotes organic nitrogen from the amino acids provided as organic carbon substrates). Circles indicate the percentage of supplied nitrogen converted to ammonium. If the amount of ammonium produced did not exceed the amount of organic nitrogen provided, it most likely originated from the degradation of organic nitrogen supplied in the medium. This was confirmed by the detection of nitrogen $\left(\mathrm{N}_{2}\right)$ as the main product. Nitrite and nitrate concentrations were $<0.5 \mu \mathrm{M}$ and $<10 \mu \mathrm{M}$, respectively (except in the "feast and famine" incubations) and electron donors were always present in a slight excess. SD $(n=3)$ values did not exceed symbol diameters.
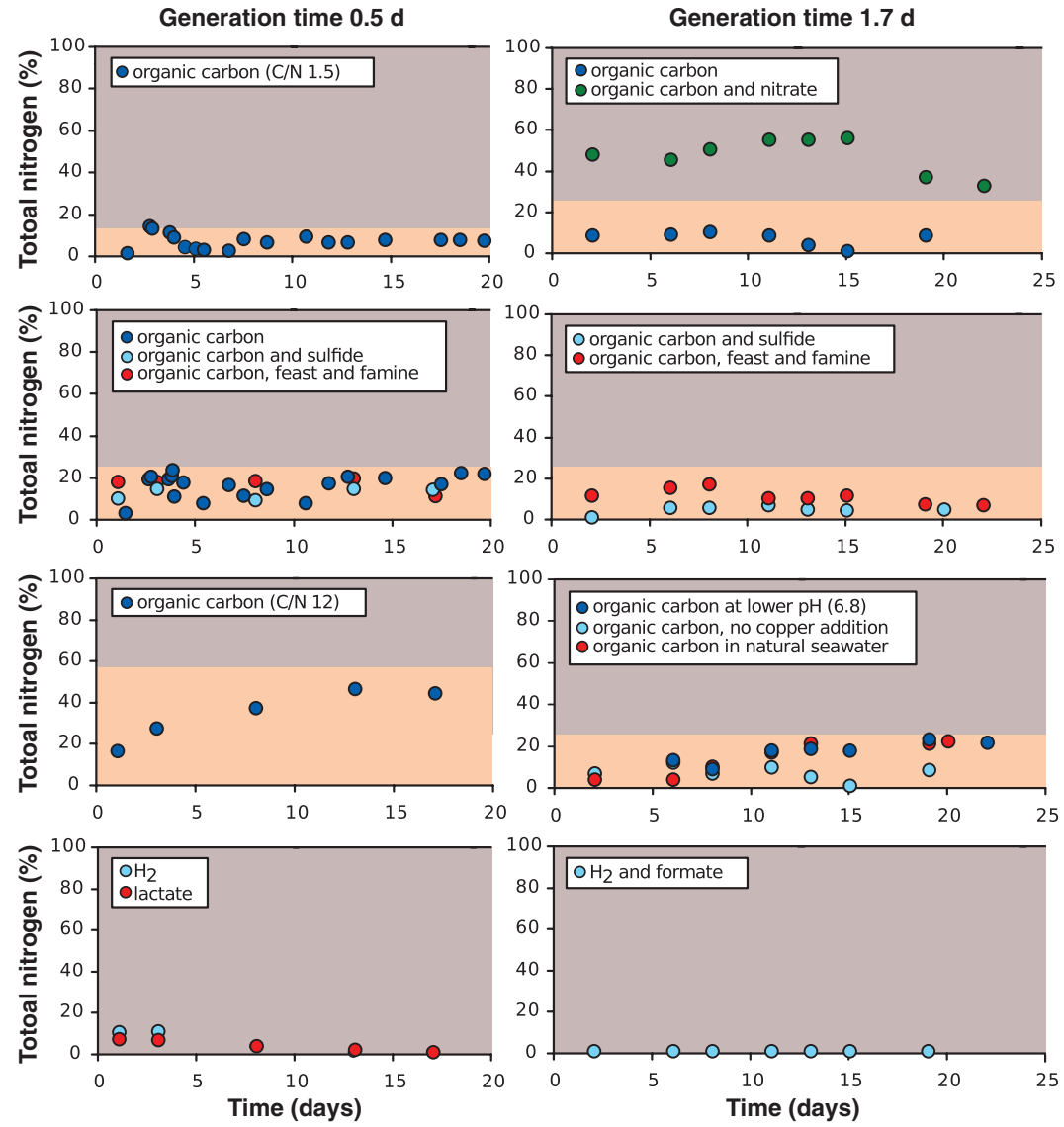

Fig. 2. Prevalence of ammonification and denitrification as a function of generation time. (A) Generation time applied. (B) Prevalence of ammonification (A) and denitrification (D) as shown by conversion of nitrogen supplied in the form of nitrate (shaded bronze area) and amino acids (shaded apricot) into ammonium (green circles; SD, $n=3$, plotted as shaded green area) and $\mathrm{N}_{2}$ (brown diamonds). (C) Relative abundance estimates of nitrate-respiring populations in metagenomes. Nitrite and nitrate concentrations remained below $0.5 \mu \mathrm{M}$ and $10 \mu \mathrm{M}$, respectively. *Bin $\mathrm{H}$ only participated in nitrate reduction to nitrite.

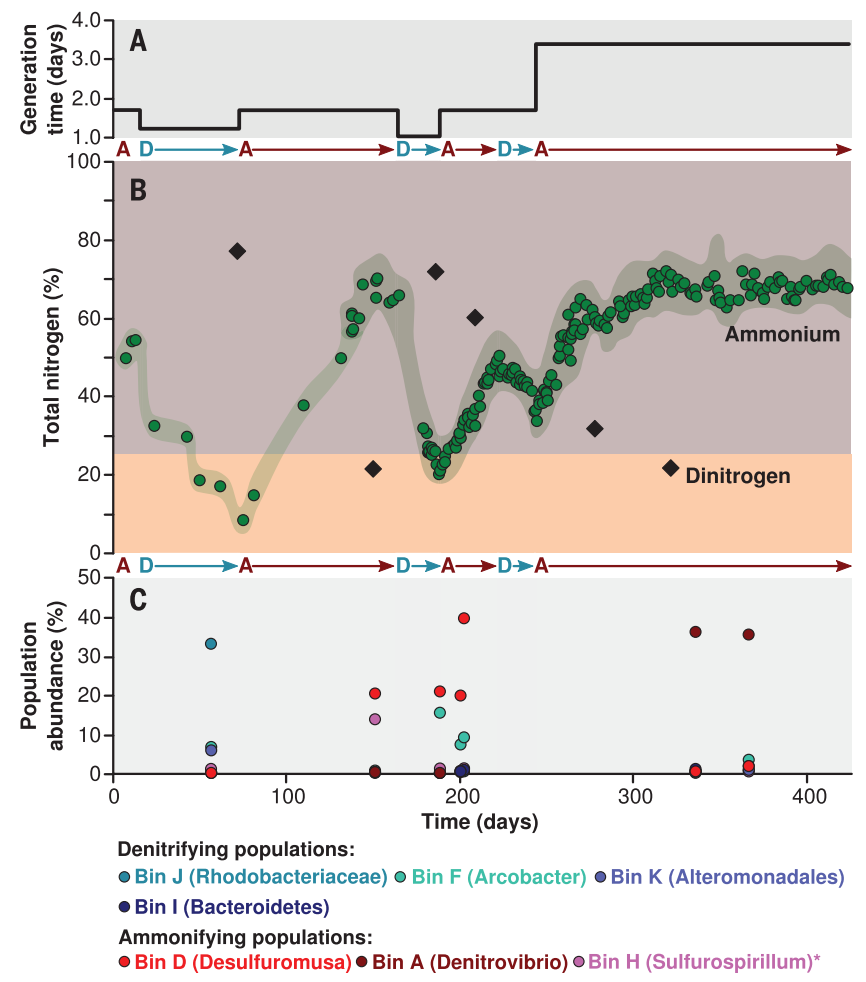


Table 1. Potential rates for nitrate and nitrite reduction coupled to the oxidation of different electron donors, and apparent activation energies $E_{\mathrm{a}}$ and Q10 coefficients for denitrification and ammonification. n.d., not determined.

\begin{tabular}{|c|c|c|c|}
\hline Electron acceptor & $\begin{array}{l}\text { Electron } \\
\text { donor }\end{array}$ & $\begin{array}{c}\text { Denitrification } \\
\left(\mathrm{nmol} \mathrm{min}^{-1} \mathrm{mg}^{-1} \text { protein }\right. \\
\end{array}$ & $\begin{array}{c}\text { Ammonification } \\
\left(\mathrm{nmol} \mathrm{min}^{-1} \mathrm{mg}^{-1} \text { protein }{ }^{-1}\right)\end{array}$ \\
\hline $\begin{array}{l}\text { Nitrate } \\
\text { Nitrate } \\
\text { Nitrite } \\
\text { Nitrite } \\
\text { Nitrite } \\
\text { Nitrite }\end{array}$ & $\begin{array}{l}\text { Organic carbon } \\
\text { Sulfide } \\
\text { Organic carbon } \\
\text { Sulfide } \\
\text { Acetate } \\
\text { Acetate + sulfide }\end{array}$ & $\begin{array}{l}61 \pm 5 \\
76 \pm 5 \\
54 \pm 5 \\
36 \pm 1 \\
25 \pm 5 \\
71 \pm 3\end{array}$ & $\begin{array}{l}48 \pm 1 \\
65 \pm 1 \\
26 \pm 1 \\
30 \pm 1 \\
24 \pm 2 \\
33 \pm 2\end{array}$ \\
\hline $\begin{array}{l}E_{a} \text { (nitrate, } \mathrm{kJ} / \mathrm{mol} \text { ) } \\
E_{a} \text { (nitrite, } \mathrm{kJ} / \mathrm{mol} \text { ) } \\
\text { Q10 (nitrate) } \\
\text { Q10 (nitrite) }\end{array}$ & & $\begin{array}{l}23 \\
\text { n.d. } \\
1.4 \\
\text { n.d. }\end{array}$ & $\begin{array}{l}28 \\
34 \\
1.5 \\
1.6\end{array}$ \\
\hline
\end{tabular}

communities with metagenomic sequencing at different time points (Fig. 2). Metagenomic reads were assembled into contigs, which were binned as described (18). We obtained 11 different bins, each bin constituting a provisional whole-genome sequence of a single population (fig. S1 and table S2). Each bin could also be linked to a full-length $16 S$ ribosomal RNA gene (fig. S2); together the bins accounted for $85 \%$ of all sequenced reads in all samples. Population abundances estimated from transcriptomes, proteomes, and catalyzed reporter deposition-fluorescence in situ hybridization (CARD-FISH) cell counts agreed well with the estimates based on the sequencing coverage of the bins (fig. S3 and table S2). The metabolic interactions of the different populations can be inferred from the metagenomic, transcriptomic, and proteomic data (Fig. 3 and table S3).

The results suggested that denitrification was mainly performed by a population affiliated to Rhodobacteraceae (bin J) on or around day 54, and by a population related to Arcobacter (bin F) on or around day 189. Ammonification appeared to be mainly performed by a population related to Desulfuromusa (bin D) on or around day 152, and by a population related to Denitrovibrio (bin A) on or around day 336. The Desulfuromusa population showed high transcriptional activity of the $n r f A H$ operon (encoding the pentaheme cytochrome $\mathrm{c}$ nitrite reductase complex that facilitates respiratory nitrite reduction to ammonium), whereas these genes were not detected for the Denitrovibrio population (table S14). Instead, this function was presumably performed by a transcriptionally highly active octaheme cytochrome c protein [OCC (19)]. The protein (contig00200_04753) clustered together with related proteins from Deferribacterales within a group of sequences from bacteria that thrive in anoxic habitats, several of which are capable of performing nitrite reduction to ammonium (fig. S4). Elevated gene transcripts in the transcriptomic and metaproteomic data suggest that both the denitrifiers and the ammonifiers reduced nitrate to nitrite with a periplasmic nitrate reductase (NapAB) (table S14).

Proteomics and transcriptomic analyses suggested that at all time points sampled, specialized populations fermented sugars and amino

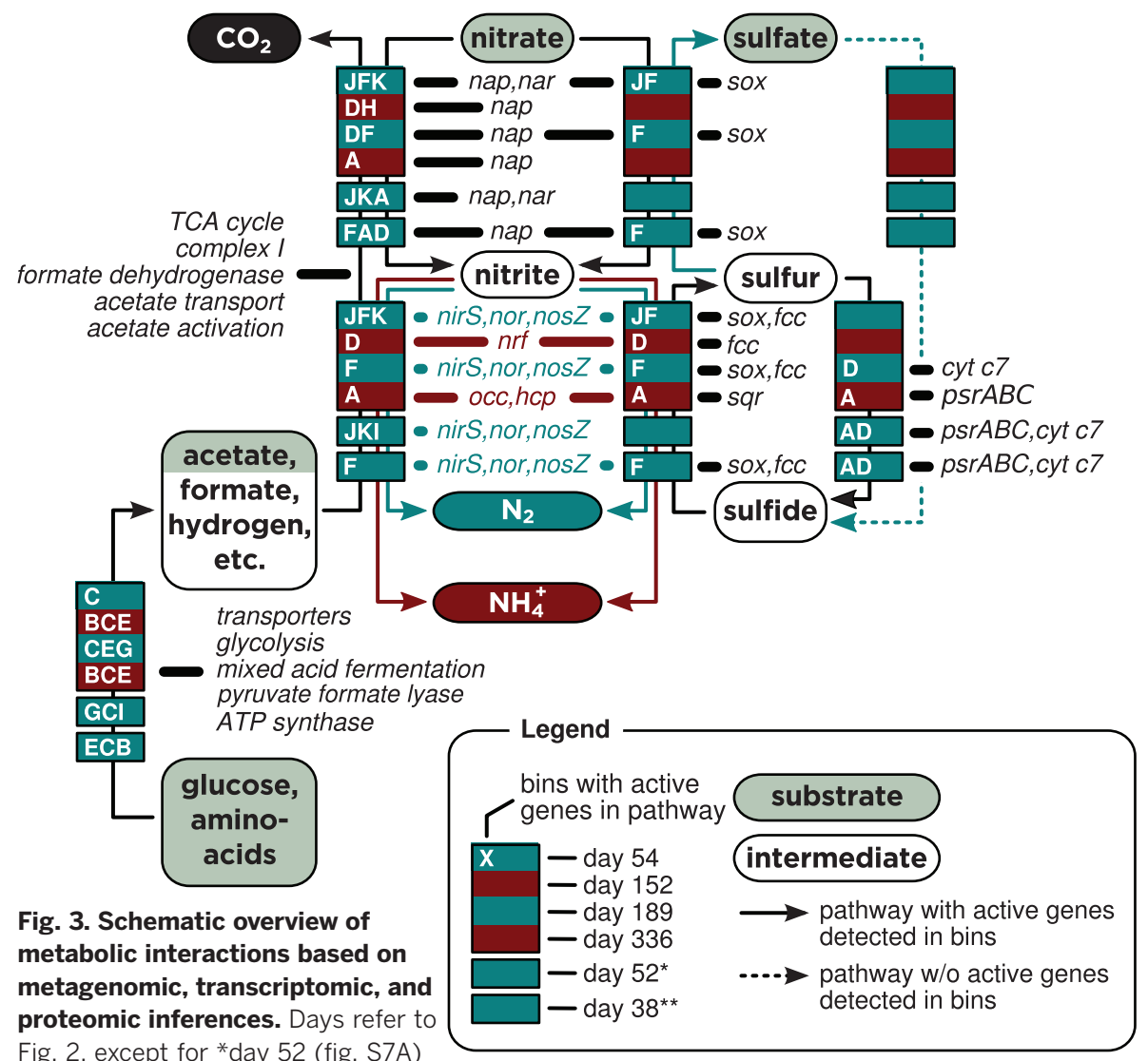

Fig. 2, except for ${ }^{*}$ day 52 (fig. S7A) and ${ }^{* *}$ day 38 (fig. S7B).

acids, as shown by the presence and activity of genes involved in mixed acid fermentation and sugar and amino acid transport in bins affiliating with different Clostridiales (bins B, C, and E) and Spirochaetales (bin G) species (Fig. 3 and table S3). All these populations were active in both the denitrifying and ammonifying stages of the experiment. These populations apparently did not participate in respiration but simply provided substrates to the nitrate-reducing populations in the form of fermentation products (e.g., acetate, formate, hydrogen). The transcriptomes and proteomes also suggested that sulfide was an ad- ditional electron donor driving nitrate respiration by the Rhodobacteraceae, Arcobacter, Sulfurospirillum (bin $\mathrm{H}$ ), and Denitrovibrio populations (Fig. 3 and table S3), and this was also confirmed experimentally (Table 1). Although no contigs of sulfate-reducing bacteria were detected in the metagenomes, incubations with $\left[{ }^{35} \mathrm{~S}\right]$ sulfate exhibited active sulfate reduction $\left(1.1 \mathrm{nmol} \mathrm{min}{ }^{-1} \mathrm{mg}\right.$ protein $^{-1}$; fig. S5). The experimental results showed that $\sim 25 \%$ of the nitrate was respired via a socalled "cryptic" sulfur cycle (20).

Transcriptomics and proteomics together revealed that the metabolic interactions between 
the populations were stable and essentially independent of generation time (Fig. 3). Denitrifiers and ammonifiers competed for the same substrates and used the same enzyme for nitrate reduction $(\mathrm{Nap} A B)$ and therefore should have similar affinities for nitrate. This means that the observed selective force of microbial generation time most likely acted on a slight difference in substrate affinity for nitrite, the branching point of the two pathways. Even though specific enzyme affinities have been determined for isolated nitrite reductases, their in vivo affinities are unknown. Apparent substrate affinities are directly proportional to potential rates, and we observed large differences between the potential rates of nitrite reduction of the two pathways, especially when multiple electron donors were provided (Table 1). For ammonification, the rates were lower than for denitrification, even leading to the transient accumulation of nitrite during ammonification of nitrate, which was not observed during denitrification (fig. S6). Lower rates with multiple electron donors might be explained by a bottleneck in electron supply to the nitrite reductases of ammonification. These require six electrons per nitrite, versus only a single electron for denitrification.

In all experiments reported so far, nitrate or nitrite were in limited supply in the presence of a slight excess of carbon substrates. According to previous studies (2-5), carbon limitation in the presence of excess nitrate should favor denitrification. To investigate this idea in the present experimental context, we performed a continuousculture incubation under carbon limitation with excess nitrate. A shorter generation time and a supply of nitrite instead of nitrate were tested in two parallel control experiments. In all three cases, denitrification became the prevalent pathway (fig. S7), confirming the importance of carbon/ nitrogen ratio in addition to generation time and supply of nitrite relative to nitrate. Finally, we investigated the potential effect of temperature on the competition by determining the apparent activation energy $\left(E_{\mathrm{a}}\right)$ and the Q10 temperature coefficient for both processes (Table 1 and fig. S8). The calculated coefficients were not significantly different $\left(F_{2,18}=1.93, P=0.174\right)$; thus, we were not able to reproduce a previously observed effect of temperature on the competition between two bacterial isolates (9).

Generation time, nitrate versus nitrite supply, and carbon/nitrogen ratio completely and reproducibly explained the fate of nitrate in 21 experimental trials with a microbial community sampled from a marine tidal flat. For these factors to hold such strong selective pressure, it is conceivable that the conditions favored the selected populations for reasons that are unrelated to nitrate respiration and cannot be extrapolated to other habitats. However, the combined results show that denitrifying and ammonifying populations were competing for the same electron donors (mainly organic acids, formate, hydrogen, and sulfide; Fig. 3) provided by the same fermentative populations. Further, selective pressure of $\mathrm{pH}$, copper, presence of sulfide, supply of fermentation products, natural seawater and temperature could be ruled out on the basis of independent experiments (Fig. 1 and fig. S8). Instead, the results suggest that the selective force acted directly on the nitrite reductases. A slightly higher apparent affinity for nitrite of the cytochrome $c d_{1}$ nitrite reductases of denitrification would explain the observed higher fitness of denitrification with nitrite as the electron acceptor and at shorter generation times. With nitrate, when the generation time is short, NrfA/OCC cannot keep up with the nitrate reductase (fig. S6) and denitrification prevails. At longer generation times, NrfA/OCC keeps up kinetically, its stoichiometric advantage with excess electrons (2) pays off, and ammonification outcompetes denitrification.

\section{REFERENCES AND NOTES}

1. D. Fowler et al., Philos. Trans. R. Soc. B 368, 20130164 (2013).

2. J. M. Tiedje, A. J. Sexstone, D. D. Myrold, J. A. Robinson, Antonie Leeuwenhoek 48, 569-583 (1983).

3. T. O. Strohm, B. Griffin, W. G. Zumft, B. Schink, Appl. Environ. Microbiol. 73, 1420-1424 (2007).

4. A. E. Tugtas, S. G. Pavlostathis, Biotechnol. Bioeng. 98. 756-763 (2007).

5. C. S. Schmidt, D. J. Richardson, E. M. Baggs, Soil Biol. Biochem 43. 1607-1611 (2011).

6. L. F. Dong et al., Appl. Environ. Microbiol. 75, 3171-3179 (2009).

7. L. F. Dong et al., Limnol. Oceanogr. 56, 279-291 (2011).

8. J. C. Akunna, C. Bizeau, R. Moletta, Water Res. 27, 1303-1312 (1993)

9. B. G. Ogilvie, M. Rutter, D. B. Nedwell, FEMS Microbiol. Ecol. 23, 11-22 (1997).
10. R. C. Brunet, L. J. Garcia-Gil, FEMS Microbiol. Ecol. 21, 131-138 (1996).

11. L. Mazéas et al.. Rapid Commun. Mass Spectrom. 22 1746-1750 (2008)

12. A. Behrendt, D. de Beer, P. Stief, Biogeosciences 10, 8065-8101 (2013)

13. See supplementary materials on Science Online

14. H. Gao et al., Limnol. Oceanogr. 57, 185-198 (2012).

15. S. B. Joye, J. T. Hollibaugh, Science 270, 623-625 (1995).

16. D. Bru et al., ISME J. 5. 532-542 (2011).

17. S. Vieira-Silva, E. P. C. Rocha, PLOS Genet. 6, e1000808 (2010) 18. A. Hanke et al., Front. Microbiol. 5, 231 (2014).

\section{ACKNOWLEDGMENTS}

Supported by European Research Council (ERC) starting grant MASEM 242635 (M.S.); ERC grant StG 306933 (J.S.G.); research incentive funds from the University of North Carolina at Charlotte, NSF grant EF-0541797, and a distinguished fellowship at the State Key Laboratory of Marine Environmental Science at Xiamen University, China (M.G.K.); the German Federal State Nordrhein-Westfalen; and the Max Planck Society. We thank I. Kattelmann and R. Vahrenhorst for their support in metagenomic sequencing and analysis, and T. Hargesheimer for CARD-FISH analysis. Raw sequencing data are publicly available under accession numbers SAMN02566827-30, SAMN02592751, SAMN02584197-8 (http://trace.ncbi.nlm.nih.gov/Traces/sra), JDSF00000000, JDSG00000000, and JDSH00000000 (www.ncbi.nlm.nih.gov/bioproject/231836).

\section{SUPPLEMENTARY MATERIALS}

www.sciencemag.org/content/345/6197/676/suppl/DC1 Materials and Methods

Figs. S1 to S8

Tables S1 to S14

References (19-30)

28 March 2014; accepted 26 June 2014

10.1126/science. 1254070

\title{
25-Hydroxycholesterol suppresses interleukin-1-driven inflammation downstream of type I interferon
}

\author{
Andrea Reboldi, ${ }^{1}$ Eric V. Dang, ${ }^{1}$ Jeffrey G. McDonald, ${ }^{2}$ Guosheng Liang, ${ }^{2}$ \\ David W. Russell, ${ }^{2}$ Jason G. Cyster ${ }^{1 *}$
}

Type I interferon (IFN) protects against viruses, yet it also has a poorly understood suppressive influence on inflammation. Here, we report that activated mouse macrophages lacking the IFN-stimulated gene cholesterol 25-hydroxylase (Ch25h) and that are unable to produce the oxysterol 25-hydroxycholesterol (25-HC) overproduce inflammatory interleukin-1 (IL-1) family cytokines. 25-HC acts by antagonizing sterol response element-binding protein (SREBP) processing to reduce $I I 1 b$ transcription and to broadly repress IL-1-activating inflammasomes. In accord with these dual actions of 25-HC, Ch25h-deficient mice exhibit increased sensitivity to septic shock, exacerbated experimental autoimmune encephalomyelitis, and a stronger ability to repress bacterial growth. These findings identify an oxysterol, $25-\mathrm{HC}$, as a critical mediator in the negative-feedback pathway of IFN signaling on IL-1 family cytokine production and inflammasome activity.

A s well as having potent antiviral activity, type I interferon (IFN) has a suppressive influence on immunity, an action that helps prevent uncontrolled inflammation and that underlies its utility in treatment of certain autoimmune diseases, such as multiple sclerosis (1-4). This suppressive action also con- tributes to the increased propensity for bacterial infection after viral infection $(1,2)$. A central facet of the IFN-mediated suppressive effect is down-regulation of inflammasome activity and interleukin-1 $\beta$ (IL-1 $\beta$ ) production $(3,5)$. However, which of the several hundred IFN-stimulated genes are responsible for these effects is poorly 